\title{
Western and Healthy Dietary Patterns and Risk of Rheumatoid Arthritis: A Case-Control Study \\ Fatemeh Rezazadeh $^{1}$, Masoumeh Akhlaghi ${ }^{* 2}$, Elham Aflaki ${ }^{3}$
}

1- Department of Clinical Nutrition, School of Nutrition and Food Sciences, Shiraz University of Medical Sciences, Shiraz, Iran

2- Nutrition Research Center, School of Nutrition and Food Sciences, Shiraz University of Medical Sciences, Shiraz, Iran

3- Department of Rheumatology, School of Medicine, Shiraz University of Medical Sciences, Shiraz, Iran

\section{A B S T R A C T}

Background and Objectives: Rheumatoid arthritis (RA) is an inflammatory autoimmune disease characterized by swelling, stiffness and pain in body joints. Based on the epidemiological studies, RA is less severe in Southern Mediterranean regions, where fish, olive oil, fruits and vegetables are consumed in great quantities.

Materials and Methods: This study was carried out in a case-control design. Totally, 100 RA patients (case group) and 100 age, gender and body mass index (BMI) healthy individuals (control group) were participated in the study. Dietary intakes were estimated using validated 160-item semi-quantitative food frequency questionnaire (FFQ). Food items of FFQ were grouped into 20 groups and dietary patterns were detected using principal component factor analysis. Associations between the adherence to dietary patterns and risk of RA were reported using logistic regression.

Results: In general, two dietary patterns were detected, including Western dietary pattern, which was high in sweet snacks, high-fat meats, refined grains, high-fat dairies and salty snacks, and healthy dietary pattern, which was high in fishes, low-fat dairies, fruits, vegetables and olives. The RA patients had a significantly higher Western diet score than that the controls had $(\mathrm{P}<0.001)$. However, no significant differences were seen in scores of healthy diet between the groups. The Western dietary pattern showed a significant positive association with RA either in the crude model $(\mathrm{OR}=2.01, \mathrm{P}<$ 0.001) or after adjustments of age, gender, education level, marital status, income, physical activity, smoking, BMI, dietary intake of vitamin $\mathrm{E}$, saturated fatty acids and polyunsaturated fatty acids $(\mathrm{OR}=4.28, \mathrm{P}<0.001)$. In contrast, the healthy dietary pattern showed an inverse significant association only after adding the highlighted parameters to the model $(\mathrm{OR}=0.55, \mathrm{P}=0.003)$.

Conclusions: This study showed a positive association between the Western dietary pattern and RA.

Keywords: Rheumatoid arthritis, RA, Dietary patterns, Shiraz

\section{Introduction}

Rheumatoid arthritis (RA) is a chronic inflammatory autoimmune disorder characterized by swelling, stiffness and pain in body joints (1). Prevalence of RA is approximately $1 \%$ worldwide (2) and $0.37 \%$ in Iran (3). The disease is more common in women than men (4). Like other autoimmune diseases, the exact cause of RA is unknown; however, genetic factors, female hormones, smoking, oxidative stress, obesity and environmental contaminants may contribute to pathogenesis of the disease (5). Roles of diets in prevention and treatment of RA have been less noticed (6). Based on the epidemiological studies, RA is less severe in Southern Mediterranean countries such as Italy and Greece, where fishes, olive oils, fruits and vegetables are consumed in relatively high quantities (7). Furthermore, a meta-analysis of few available prospective studies showed the beneficial effects of healthy diets in decreasing pains and increasing physical functions of RA patients (8). It has been reported that durations of morning stiffness and disability and C-reactive protein concentrations in RA patients are inversely correlated with healthy eating indices $(9,10)$. Fibers and whole grains, fruits, fishes and $\omega$-3-fatty acids are the most efficacious compounds in treatment of RA $(11,12)$. However, a cross-sectional study showed that most RA patients 
did not follow dietary recommendations of fruits, vegetables and whole grains and did not sufficiently restricted sodium and empty calories (9). Compared to components of healthy diets, associations between the RA and constituents of unhealthy diets (diets low in fruits and vegetables and high in saturated fats, sugar-sweetened snacks and salts) have been investigated less. In particular, no studies have assessed Western dietary patterns in RA patients. Moreover, most studies have investigated individual dietary components rather than the whole diets (11). Dietary patterns are defined as "the quantity, variety or combination of different foods and beverages in a diet and the frequency with which they are habitually consumed" (13). In fact, dietary patterns are useful tools for investigating possible links between the diets and chronic diseases because these patterns provide comprehensive views of food and nutrient consumptions (14). The aims of the current study included assessment of dietary patterns in RA patients and comparison of these dietary patterns with those of healthy age, gender and BMI-matched individuals.

\section{Materials and Methods}

Study design and participants. This study was a cross-sectional study with case-control design. The sample size was calculated based on a hypothetical odds ratio of 0.41 (15), a power of $80 \%$, a two-sided confidence interval of $5 \%$ and a case/control sample ratio of 1 . Totally, $100 \mathrm{RA}$ patients (case group) and 100 age, gender and body mass index (BMI) matched healthy individuals (control group) were participated in the study. The patients were selected from a rheumatology clinic of Shiraz University of Medical Sciences, Shiraz, Iran. Inclusion criteria included diagnosis of RA by a rheumatologist based on 2010 Rheumatoid Arthritis Classification Criteria (16), absence of other pathological conditions such as diabetes mellitus, hepatic disorders, thyroid abnormalities, cancers, food allergies and nutritional deficiencies, absence of adherence to specific diets and consumption of dietary supplements. Exclusion criteria included incomplete answers to food frequency questionnaires (FFQ) by the participants and extreme energy intake reporters (women reporting energy intake less than 600 or higher than $3500 \mathrm{kcal} \mathrm{day}^{-1}$ and men with energy intake less than 800 or higher than $4200 \mathrm{kcal} \mathrm{day}^{-1}$ ) (17). Control participants were selected from healthy individuals who attended the clinic with the patients as companions. All participants were signed written informed consents. The study was carried out based on the guidelines of the Ethics Committee of Shiraz University of Medical Sciences (approval no. 95-0184-12050).

Measurements. Anthropometric parameters, including height, weight and waist circumference, were measured and BMI was calculated as described previously (18). Physical activity was measured as metabolic equivalent task (Met-min week ${ }^{-1}$ ) using validated international physical activity questionnaires (19). Participants were asked about the time spent in sitting position. A participant was considered as current smoker if he/she smoked at least 100 cigarettes in his/her lifetime or was smoker at the time of the interview (20).

Diet assessment. An expert dietitian carried out dietary assessments using validated 160-item semiquantitative FFQ (21). The FFQ included foods with serving sizes commonly consumed by Iranians. Participants were asked to report the frequency of consumption of each food item in stated portion sizes during the previous year on a daily, weekly or monthly basis. Then, portion sizes were converted to grams, using household scales. Nutrient compositions of the consumed foods were analyzed using a modified Nutritionist IV v.3.5.2.

Statistical analysis. Food items within the FFQ were divided into 20 groups based on the similarity of their nutrient profiles. Dietary patterns were detected using principal component factor analysis with varimax rotation on the 20 food groups. Two dietary patterns were selected based on various criteria, including eigenvalues greater than 1 , rotated factor loading greater than 0.3 and a clear inflection in scree plot. The factor score for each pattern was calculated using the sum of all food groups weighted by their factor loadings. Factor scores of the individuals for adherence to each dietary pattern were calculated using Bartlett method. In each dietary pattern, higher Bartlett scores showed greater adherences while lower scores demonstrated lower adherences. Data were analyzed using SPSS Software v.21 (IBM Analytics, USA). Demographic and anthropometric data, blood pressure and dietary intakes were compared with each other between the case (RA patients) and control (healthy individuals) groups using independent $t$-test. Associations between the participant adherence to dietary patterns and risk of RA were reported using 
logistic regression. In unadjusted models, no covariates were entered in the models; however, adjustments were carried out using age and gender in Model 1, age, gender, education level, marital status, income, physical activity, smoking and BMI in Model 2 and previous parameters as well as vitamin $\mathrm{E}$, saturated fatty acids (SFA) and polyunsaturated fatty acids (PUFA) intakes in Model 3. Statistical significance was set at $\mathrm{P}<0.05$.

\section{Results}

Demographic characteristics, anthropometric measures and dietary pattern scores were compared between case and control groups (Table 1). Waist circumferences were significantly higher in RA patients, compared to those in controls $(\mathrm{P}<0.001)$.

Two dominant dietary patterns were detected. Healthy dietary pattern was rich in fishes, low-fat dairies, fruits, vegetables, olives and poultries and low in hydrogenated oils. Western dietary pattern was rich in sweet snacks, high-fat meats and meat byproducts, refined grains, high-fat dairies and salty snacks and low in whole grains (Table 2). Factor loading scores are reported in Table 2. Scores represent the contribution of each food group in the respected dietary pattern. Negative and positive signs indicate correlation directions.

Table 1. Demographic characteristics of the patients in case and control groups ${ }^{1}$

\begin{tabular}{lccc}
\hline & Case $(\mathrm{n}=100)$ & Control $(\mathrm{n}=100)$ & P value \\
\hline Age, $\mathrm{y}$ & $50.49 \pm 11.25$ & $49.18 \pm 12.55$ & 0.44 \\
Males, $\mathrm{n}(\%)$ & $15(15)$ & $15(15)$ & 1 \\
Body mass index, $\mathrm{kg} / \mathrm{m}^{2}$ & $26.19 \pm 4.00$ & $26.49 \pm 3.68$ & 0.59 \\
Waist circumference, cm & $94.82 \pm 12.69$ & $87.72 \pm 8.03$ & $<0.001$ \\
Physical activity, Met-min/week & $4.14 \pm 6.84$ & $3.63 \pm 3.87$ & 0.52 \\
Sitting, h/day & $5.00 \pm 2.41$ & $5.14 \pm 1.18$ & 0.60 \\
Smokers, $\mathrm{n}(\%)$ & $3(3)$ & $3(3)$ & 1 \\
\hline
\end{tabular}

Table 2. Factor loading scores of the dominant dietary patterns ${ }^{1}$

\begin{tabular}{|c|c|c|}
\hline Food groups & $\begin{array}{c}\text { Healthy dietary } \\
\text { pattern }\end{array}$ & $\begin{array}{c}\text { Western dietary } \\
\text { pattern }\end{array}$ \\
\hline Hydrogenated oils & -0.62 & \\
\hline Fish & 0.54 & \\
\hline Low-fat dairy & 0.52 & -0.34 \\
\hline Fruits & 0.51 & \\
\hline Non-starchy vegetables & 0.51 & \\
\hline Vegetable oils & 0.45 & 0.25 \\
\hline Olives and olive oil & 0.45 & \\
\hline Poultry & 0.45 & \\
\hline \multicolumn{3}{|l|}{ Sugar-sweetened juice } \\
\hline \multicolumn{3}{|l|}{ Nuts } \\
\hline Sweet snacks & & 0.66 \\
\hline High-fat meat and meat products & & 0.55 \\
\hline Refined grains & & 0.54 \\
\hline High-fat dairy & & 0.47 \\
\hline Salty snacks & & 0.43 \\
\hline Egg & & 0.41 \\
\hline Whole grains & & -0.32 \\
\hline Starchy vegetables & & 0.30 \\
\hline \multicolumn{3}{|l|}{ Red meat } \\
\hline Legumes & & \\
\hline
\end{tabular}


Table 3 compares dietary intakes of participants in case and control groups. Dietary intakes were not different between the two groups, except for PUFA, SFA and vitamin E. The RA patients had a significantly higher Western dietary pattern score, compared to that the controls had $(\mathrm{P}<0.001)$. However, no significant differences were seen in healthy dietary pattern mean scores between the groups.

Associations between the dietary patterns and RA were reported using logistic regression (Table 4). The healthy dietary pattern did not show a significant association with RA in crude models (unadjusted model) or after performing adjustments for age and gender (Model 1) or age, gender, education level, marital status, income, physical activity, smoking and BMI (Model 2). However, addition of dietary vitamin E, SFA and PUFA to covariates revealed a significant inverse association between the healthy dietary pattern and RA (Model 3) $(\mathrm{OR}=0.55, \mathrm{P}=0.003)$. These dietary items were selected as covariates because their intakes were significantly different between the case and control groups. The Western dietary pattern showed a significant positive association with RA either in the unadjusted model $(\mathrm{OR}=2.01, \mathrm{P}<0.001)$ or after addition of the parameters $(\mathrm{OR}=4.28, \mathrm{P}<0.001)($ Table 4$)$.

Table 3. Dietary intakes and pattern scores of the patients in case and control groups ${ }^{1}$

\begin{tabular}{lccc}
\hline & Case $(\mathrm{n}=100)$ & Control $(\mathrm{n}=100)$ & P value \\
\hline Energy, kcal/day & $1809 \pm 427.1$ & $1719 \pm 301.6$ & 0.09 \\
Protein, g/1000 kcal/day & $32.09 \pm 3.53$ & $32.46 \pm 3.11$ & 0.6 \\
Carbohydrate, g/1000 kcal/day & $150.3 \pm 10.18$ & $147.9 \pm 9.35$ & 0.08 \\
Fat, g/1000 kcal/day & $30.07 \pm 4.34$ & $30.14 \pm 3.83$ & 0.90 \\
SFA, g/1000 kcal/day & $8.77 \pm 1.58$ & $9.28 \pm 1.44$ & 0.02 \\
MUFA, g/1000 kcal/day & $9.37 \pm 1.83$ & $9.32 \pm 1.43$ & 0.80 \\
PUFA, g/1000 kcal/day & $7.07 \pm 1.54$ & $6.44 \pm 1.61$ & 0.006 \\
Cholesterol, g/1000 kcal/day & $90.98 \pm 26.63$ & $96.17 \pm 29.78$ & 0.20 \\
Fiber, g/1000 kcal/day & $2.23 \pm 0.93$ & $2.16 \pm 0.95$ & 0.58 \\
Vitamin C, mg/1000 kcal/day & $78.07 \pm 22.23$ & $81.44 \pm 26.61$ & 0.12 \\
Vitamin E, mg/1000 kcal/day & $5.26 \pm 1.21$ & $4.48 \pm 0.90$ & $<0.001$ \\
Vitamin A, $\mu \mathrm{g} / 1000 \mathrm{kcal} /$ day & $383.7 \pm 131.7$ & $416.2 \pm 231.5$ & 0.22 \\
Carotenoids, $\mu \mathrm{g} / 1000 \mathrm{kcal} /$ day & $274.4 \pm 98.39$ & $258.2 \pm 108.6$ & 0.27 \\
Sodium, g/1000 kcal/day & $1.51 \pm 2.84$ & $1.42 \pm 1.91$ & 0.01 \\
Potassium, mg/1000 kcal/day & $1.65 \pm 2.43$ & $1.70 \pm 2.80$ & 0.16 \\
Healthy dietary pattern score & $-0.071 \pm 0.95$ & $0.042 \pm 1.01$ & 0.42 \\
Western dietary pattern score & $0.26 \pm 0.91$ & $-0.30 \pm 0.89$ & $<0.001$
\end{tabular}

\footnotetext{
${ }^{1}$ Data are expressed as mean \pm SD (standard deviation) or n; MUFA, monounsaturated fatty acids; PUFA, polyunsaturated fatty acids; SFA, saturated fatty acids
}

Table 4. Associations between the adherence to dietary patterns and RA $(n=200)^{1}$

\begin{tabular}{lcccccc}
\hline & \multicolumn{3}{c}{ Healthy dietary pattern } & \multicolumn{3}{c}{ Western dietary pattern } \\
\cline { 2 - 7 } & OR & $95 \%$ CI & P value & OR & $95 \%$ CI & P value \\
\hline Unadjusted model & 0.92 & $0.70,1.22$ & 0.56 & 2.01 & $1.43,2.81$ & $<0.001$ \\
Adjusted model 1 $^{2}$ & 0.91 & $0.68,1.20$ & 0.49 & 2.78 & $1.83,4.19$ & $<0.001$ \\
Adjusted model 2 $^{3}$ & 0.86 & $0.64,1.15$ & 0.32 & 2.95 & $1.91,4.55$ & $<0.001$ \\
Adjusted model 3 $^{4}$ & 0.55 & $0.37,0.82$ & 0.003 & 4.28 & $2.30,7.97$ & $<0.001$ \\
\hline
\end{tabular}

\footnotetext{
${ }^{1}$ Associations were reported using linear regression; ${ }^{2}$ Model 1 was adjusted for age and gender; ${ }^{3}$ Model 2 was adjusted for age, gender, education level,
} marital status, income, physical activity, smoking and BMI; ${ }^{4}$ Model 3 was adjusted for previous confounders as well as PUFA, SFA and vitamin E 


\section{Discussion}

The present case-control study showed that RA included a strong positive association with the Western dietary pattern and an inverse association with the healthy dietary pattern; however, the latter was significant only after performing adjustments for intakes of vitamin E, SFA and PUFA. The association between dietary patterns and RA has rarely been investigated. In contrast, previous studies mostly investigated associations between the diet components and RA. Ingredients of the healthy dietary pattern were inversely correlated with RA risk. Cerhan et al. in a 10-year prospective cohort study on women aged 55-69 years found that consumption of diets high in fruits and cruciferous vegetables and intake of supplemental zinc and antioxidants might prevent RA (22). Pattison et al., following a nested case-control study, reported a positive association between the vitamin $\mathrm{C}$ intake and inflammatory polyarthritis (23). Moreover, 10-year prospective cohort (24) and case-control (25) studies showed that consumption of $\geq 1$ serving fish week $^{-1}$ was associated to 20-29\% decreases in RA risk, compared to that consumption of $<1$ serving fish week $^{-1}$ did. This decrease following the consumption of $\geq 1$ serving fish week ${ }^{-1}$ was concordant with a lower risk of RA following the consumption of $>0.21$ g $\omega$-3-fatty acids day ${ }^{-1}$ (24). Similarly, an inverse association was observed between the olive oils or cooked vegetables and RA risk in a case-control study in Greece (15). Protections against RA development have been reported in regular consumptions of mushrooms, citrus fruits and dairy products (26). Ingredients of the Western dietary pattern have been associated with RA risk. A positive association was observed between the consumption of red meats, meat byproducts and total proteins and the risk of inflammatory polyarthritis (IPA) (27). Furthermore, ingredients of healthy and Western dietary patterns are correlated with RA activity. A case-control study showed a correlation between the monounsaturated fatty acids (MUFA) intake and suppression of RA activity (28). Clinical trials have documented decreases in inflammatory responses and disease activities following sodium restriction (29) or ingestion of fish oil $\omega$-3-fatty acids (30). Despite the highlighted studies, associations between the diet and RA risk were not seen in a prospective cohort with a relatively short duration (5.3 years of follow-ups) and limited incident cases (31). Similarly, a longer term (18 years) prospective cohort with a greater number of RA cases found no significant associations between the Mediterranean dietary pattern and RA risk; possibly due to the assessment of Mediterranean diet adherence by an unsuitable score, especially for the detection of between-individual variations for nuts and MUFA/SFA ratios (32).

Mechanisms of the beneficial effects of healthy diets against RA have partially been explained. By increasing $\omega-3 / \omega-6$ ratio of the fatty acids in diets, production of pro-inflammatory is suppressed while production of anti-inflammatory eicosanoids is improved (33). Moreover, $\omega-3$-fatty acids at doses > $2.7 \mathrm{~g} \mathrm{day}^{-1}$ for more than three months can decrease non-steroidal anti-inflammatory drug consumptions in patients; as indicated by a meta-analysis of randomized controlled trials (34). The antiinflammatory effects of fruits and vegetables are largely due to the high levels of antioxidants (35) and polyphenols (36) that block the induction of inflammatory cytokines by inhibiting the activation of nuclear factor kappa B (NF-kB) (35). The protective effect of olive oil against RA has been suggested via prevention of metalloproteinase-3 and degradation of cartilage matrices (37). Polyphenol extracts of the extra-virgin olive oil have been shown to inhibit c-Jun $\mathrm{N}$-terminal kinase, p38 and signal transducer and activator of transcription-3 and to decrease NF- $\kappa B$ translocation, leading to downregulations of arthritic processes (38). In contrast, the Western dietary pattern includes ingredients that instigate inflammatory processes. For example, the high SFA in Western dietary pattern activates toll-like receptor mediated inflammatory pathways through oxidative modifications of low-density lipoproteins (39). Salt, another ingredient of the Western dietary pattern, enhances differentiation of interleukin-17 producing helper T-cells, which are responsible for the development of arthritis (29). Whole grains in the healthy dietary pattern decrease inflammations $(40,41)$, while sweets and refined grains in the Western dietary pattern causes high glycemic loads, leading to persistent hyperglycemia, production of advanced glycation products, insulin resistance and initiation of inflammatory cascade (42-44). The positive association between the egg consumption and RA risk that is found in the current study has not been 
reported by the previous studies; however, current evidence indicate associations between the serum cholesterol and RA risk $(45,46)$. This association might be a resultant of the inflammatory processes caused by high serum cholesterols (47). However, the current study included strengths and limitations like most other studies. The case-control design of the study helped to detect associations between diets and RA. Detecting dietary patterns instead of assessing individual diet components was another strength which distinguished the present study from previous studies. However, the cross-sectional design of this study could not determine whether the diet was a trigger or a consequence of RA. It is worthy to use new cases of RA, an approach that allows studying roles of diets in development of the disease. There were also limitations with the FFQ method. For example, items questioned in the questionnaire were the most common foods consumed, but possibly other foods contributed to diets of people. Another limitation of the FFQ included its reliance on the participant recollection of the past year food consumption, which could result in erroneous dietary measurements.

\section{Conclusion}

In conclusion, results of this study showed a strong association between the RA and Western dietary patterns for the first time. Patients with RA were inclined towards Western dietary patterns, while healthy controls were not. Although a cause-effect correlation could not be concluded from this study, results suggest that educational efforts are necessary to rectify dietary habits in RA patients. Future studies seems necessary to investigate roles of dietary patterns in occurrence of rheumatoid arthritis.

\section{Acknowledgement}

Results of this study were extracted from a thesis written by Ms. Fatemeh Rezazadeh. This study was financially supported by Shiraz University of Medical Sciences, grant number 95-01-84-12050. The authors wish to thank Mr. Argasi at the Research Consultation Center (RCC), Shiraz University of Medical Sciences, for his valuable assistance in editing this manuscript.

\section{Ethical statement}

This study was carried out based on the guidelines by the Ethics Committee of Shiraz University of Medical Sciences (approval no. 95-01-84-12050).

\section{Financial disclosure}

The authors declared no financial interest.

\section{Funding/Support}

This study was financially supported by Shiraz University of Medical Sciences (grant no. 95-01-8412050).

\section{References}

1. Calabresi E, Petrelli F, Bonifacio AF, Puxeddu I, Alunno A. One year in review 2018: pathogenesis of rheumatoid arthritis. Clin Exp Rheumatol. 2018;36(2):175-184.

2. Gibofsky A. Overview of epidemiology, pathophysiology, and diagnosis of rheumatoid arthritis. Am J Manag Care. 2012;18(13 Suppl):S295302 .

3. Davatchi F, Sandoughi M, Moghimi N, Jamshidi AR, Tehrani Banihashemi A, Zakeri Z, Sadeghi Abdollahi B. Epidemiology of rheumatic diseases in Iran from analysis of four COPCORD studies. Int $\mathbf{J}$ Rheum Dis. 2016 Nov;19(11):1056-1062. doi: 10.1111/1756-185X.12809.

4. Favalli EG, Biggioggero M, Crotti C, Becciolini A, Raimondo MG, Meroni PL. Sex and management of rheumatoid arthritis. Clin Rev Allergy Immunol. 2018 Jan 26. doi: 10.1007/s12016-018-8672-5. [Epub ahead of print]

5. Srivastava S, Patel S, Singh D, Singh MR. Rationalized insights on causes of rheumatoid arthritis in the elderly and women: special emphasis on treatment strategies. Crit Rev Ther Drug Carrier Sys. 2017;34(2):97-147.

6. Tedeschi SK, Costenbader KH. Is there a role for diet in the therapy of rheumatoid arthritis? Curr Rheumatol Rep. 2016;18(5):23. doi: 10.1007/s11926016-0575-y.

7. Pattison DJ, Harrison RA, Symmons DP. The role of diet in susceptibility to rheumatoid arthritis: a systematic review. J Rheumatol. 2004;31(7):1310-9.

8. Forsyth C, Kouvari M, D'Cunha NM, Georgousopoulou EN, Panagiotakos DB, Mellor DD, Kellett J, Naumovski N. The effects of the Mediterranean diet on rheumatoid arthritis prevention and treatment: a systematic review of human prospective studies. Rheumatol Int. 2018;38(5):737747. doi: 10.1007/s00296-017-3912-1.

9. Berube LT, Kiely M, Yazici Y, Woolf K. Diet quality of individuals with rheumatoid arthritis using the Healthy Eating Index (HEI)-2010. Nutr Health. 2017;23(1):17-24. doi: 10.1177/0260106016688223.

10. Grimstvedt ME, Woolf K, Milliron BJ, Manore MM. Lower Healthy Eating Index-2005 dietary quality scores in older women with rheumatoid arthritis v. healthy controls. Public Health Nutr. 2010;13(8):1170-7. 
11. Khanna S, Jaiswal KS, Gupta B. Managing rheumatoid arthritis with dietary interventions. Front Nutr. 2017;4:52. doi: 10.3389/fnut.2017.00052.

12. Badsha $\mathrm{H}$. Role of diet in influencing rheumatoid arthritis disease activity. Open Rheumatol J. 2018;12:19-28. doi: 10.2174/1874312901812010019.

13. Reedy J, Subar AF, George SM, Krebs-Smith SM. Extending methods in dietary patterns research. nutrients. 2018 May 7;10(5). pii: E571. doi: 10.3390/nu10050571.

14. Jacques PF, Tucker KL. Are dietary patterns useful for understanding the role of diet in chronic disease? Am J Clin Nutr. 2001 Jan;73(1):1-2.

15. Linos A, Kaklamani VG, Kaklamani E, Koumantaki Y, Giziaki E, Papazoglou S, Mantzoros CS. Dietary factors in relation to rheumatoid arthritis: a role for olive oil and cooked vegetables? Am J Clin Nutr. 1999;70(6):1077-82.

16. Aletaha D, Neogi T, Silman AJ, Funovits J, Felson DT, Bingham CO 3rd, et al. 2010 Rheumatoid arthritis classification criteria: an American College of Rheumatology/European League Against Rheumatism collaborative initiative. Arthritis Rheum. 2010;62(9):2569-81. doi: 10.1002/art.27584.

17. Willett W. Nutritional epidemiology. Oxford University Press, New York. 1998.

18. Akhlaghi M, Kamali M, Dastsouz F, Sadeghi $\mathrm{F}$, Amanat S. Increased waist-to-height ratio may contribute to agerelated increase in cardiovascular risk factors. Int $\mathrm{J}$ Prev Med. 2016;7:68.

19. Lee PH, Macfarlane DJ, Lam TH, Stewart SM. Validity of the international physical activity questionnaire short form (IPAQ- SF): A systematic review. Int J Behav Nutr Phys Act. 2011;8:115.

20. Centers for Disease Control and Prevention, "Cigarette smoking among adults-United States, 1992, and changes in the definition of current cigarette smoking," Morbidity and Mortality Weekly Report, vol. 43, no. 19, pp. 342-346, 1994.

21. Esfahani FH, Asghari G, Mirmiran P, Azizi F. Reproducibility and relative validity of food group intake in a food frequency questionnaire developed for the Tehran Lipid and Glucose Study. J Epidemiol. 2009;20(2):150-8.

22. Cerhan JR, Saag KG, Merlino LA, Mikuls TR, Criswell LA. Antioxidant micronutrients and risk of rheumatoid arthritis in a cohort of older women. Am J Epidemiol. 2003;157(4):345-54.

23. Pattison DJ, Symmons DP, Young A. Does diet have a role in the aetiology of rheumatoid arthritis? Proc Nutr Soc. 2004;63(1):137-43.

24. Di Giuseppe D, Wallin A, Bottai M, Askling J, Wolk A. Long-term intake of dietary long-chain n-3 polyunsaturated fatty acids and risk of rheumatoid arthritis: a prospective cohort study of women. Ann Rheum Dis. 2014;73(11):1949-53.

25. Rosell M, Wesley AM, Rydin K, Klareskog L, Alfredsson L; EIRA study group. Dietary fish and fish oil and the risk of rheumatoid arthritis. Epidemiology. 2009;20(6):896-901. doi: 10.1097/EDE.0b013e3181b5f0ce.

26. He J, Wang Y, Feng M, Zhang X, Jin YB, Li X, et al. Dietary intake and risk of rheumatoid arthritis-a cross section multicenter study. Clin Rheumatol. 2016;35(12):2901-2908.

27. Pattison DJ, Symmons DP, Lunt M, Welch A, Luben R, Bingham SA, Khaw KT, Day NE, Silman AJ. Dietary risk factors for the development of inflammatory polyarthritis: evidence for a role of high level of red meat consumption. Arthritis Rheum. 2004;50(12):3804-12.

28. Matsumoto Y, Sugioka Y, Tada M, Okano T, Mamoto K, Inui $\mathrm{K}$, Habu $\mathrm{D}$, Koike $\mathrm{T}$. Monounsaturated fatty acids might be key factors in the Mediterranean diet that suppress rheumatoid arthritis disease activity: The TOMORROW study. Clin Nutr. 2018;37(2):675-680. doi: 10.1016/j.clnu.2017.02.011.

29. Scrivo R, Massaro L, Barbati C, Vomero M, Ceccarelli F, Spinelli FR, Riccieri V, Spagnoli A, Alessandri C, Desideri G, Conti F, Valesini G. The role of dietary sodium intake on the modulation of $\mathrm{T}$ helper 17 cells and regulatory $\mathrm{T}$ cells in patients with rheumatoid arthritis and systemic lupus erythematosus. PLoS One. 2017;12(9):e0184449. doi: 10.1371/journal.pone.0184449.

30. Berbert AA, Kondo CR, Almendra CL, Matsuo T, Dichi I. Supplementation of fish oil and olive oil in patients with rheumatoid arthritis. Nutrition. 2005;21(2):131-6.

31. Pedersen M, Stripp C, Klarlund M, Olsen SF, Tjønneland AM, Frisch M. Diet and risk of rheumatoid arthritis in a prospective cohort. J Rheumatol. 2005;32(7):1249-52.

32. Hu Y, Costenbader KH, Gao X, Hu FB, Karlson EW, $\mathrm{Lu}$ B. Mediterranean diet and incidence of rheumatoid arthritis in women. Arthritis Care Res (Hoboken). 2015;67(5):597-606. doi: 10.1002/acr.22481.

33. Wall R, Ross RP, Fitzgerald GF, Stanton C. Fatty acids from fish: the anti-inflammatory potential of long-chain omega-3 fatty acids. Nutr Rev. 2010;68(5):280-9.

34. Lee YH, Bae SC, Song GG. Omega-3 polyunsaturated fatty acids and the treatment of rheumatoid arthritis: a meta-analysis. Arch Med Res. 2012;43(5):356-62.

35. Serafini M, Peluso I. Functional foods for health: the interrelated antioxidant and anti-inflammatory role of 
fruits, vegetables, herbs, spices and cocoa in humans. Curr Pharm Des. 2016;22(44):6701-6715. doi: $10.2174 / 1381612823666161123094235$.

36. Joseph SV, Edirisinghe I, Burton-Freeman BM. Fruit polyphenols: a review of anti-inflammatory effects in humans. Crit Rev Food Sci Nutr. 2016;56(3):419-44. doi: 10.1080/10408398.2013.767221.

37. Rosillo MA, Sánchez-Hidalgo M, Sánchez-Fidalgo S, Aparicio-Soto M, Villegas I, Alarcón-de-la-Lastra C. Dietary extra-virgin olive oil prevents inflammatory response and cartilage matrix degradation in murine collagen-induced arthritis. Eur J Nutr. 2016;55(1):315-25. doi: 10.1007/s00394-015-0850-0.

38. Rosillo MÁ, Alcaraz MJ, Sánchez-Hidalgo M, Fernández-Bolaños JG, Alarcón-de-la-Lastra C1, Ferrándiz ML. Anti-inflammatory and joint protective effects of extra-virgin olive-oil polyphenol extract in experimental arthritis. J Nutr Biochem. 2014;25(12):1275-81.

39. Rocha DM, Caldas AP, Oliveira LL, Bressan J, Hermsdorff HH. Saturated fatty acids trigger TLR4mediated inflammatory response. Atherosclerosis. 2016;244:211-5.

40. Priebe MG, Wang H, Weening D, Schepers $M$, Preston T, Vonk RJ. Factors related to colonic fermentation of nondigestible carbohydrates of a previous evening meal increase tissue glucose uptake and moderate glucose-associated inflammation. Am J Clin Nutr. 2010;91(1):90-7.

41. Qi L, Hu FB. Dietary glycemic load, whole grains, and systemic inflammation in diabetes: the epidemiological evidence. Curr Opin Lipidol. 2007;18(1):3-8.

42. Hu Y, Costenbader KH, Gao X, Hu FB, Karlson EW, $\mathrm{Lu}$ B. Mediterranean diet and incidence of rheumatoid arthritis in women. Arthritis Care Res (Hoboken). 2015;67(5):597-606. doi: 10.1002/acr.22481.

43. Liu S, Manson JE, Buring JE, Stampfer MJ, Willett WC, Ridker PM. Relation between a diet with a high glycemic load and plasma concentrations of highsensitivity C-reactive protein in middle-aged women. Am J Clin Nutr. 2002;75(3):492-8.

44. Chung CP, Oeser A, Solus JF, Gebretsadik T, Shintani A, Avalos I, Sokka T, Raggi P, Pincus T, Stein CM. Inflammation-associated insulin resistance: differential effects in rheumatoid arthritis and systemic lupus erythematosus define potential mechanisms. Arthritis Rheum. 2008;58(7):2105-12.

45. Turesson C, Bergström U, Pikwer M, Nilsson JA, Jacobsson LT. High serum cholesterol predicts rheumatoid arthritis in women, but not in men: a prospective study. Arthritis Res Ther. 2015;17:284. doi: 10.1186/s13075-015-0804-1.

46. Heliövaara M, Aho K, Knekt P, Reunanen A, Aromaa A. Serum cholesterol and risk of rheumatoid arthritis in a cohort of 52800 men and women. $\mathrm{Br}$ J Rheumatol. 1996;35(3):255-7.

47. Catapano AL, Pirillo A, Norata GD. Vascular inflammation and low-density lipoproteins: is cholesterol the link? A lesson from the clinical trials. Br J Pharmacol. 2017;174(22):3973-3985. 